This item was submitted to Loughborough's Research Repository by the author.

Items in Figshare are protected by copyright, with all rights reserved, unless otherwise indicated.

\title{
The simulation of aerial movement-I. The determination of orientation angles from film data
}

PLEASE CITE THE PUBLISHED VERSION

PUBLISHER

(C) Elsevier

VERSION

AM (Accepted Manuscript)

LICENCE

CC BY-NC-ND 4.0

\section{REPOSITORY RECORD}

Yeadon, Maurice R.. 2019. "The Simulation of Aerial Movement-i. The Determination of Orientation Angles from Film Data". figshare. https://hdl.handle.net/2134/5298. 
This item was submitted to Loughborough's Institutional Repository (https://dspace.lboro.ac.uk/) by the author and is made available under the following Creative Commons Licence conditions.

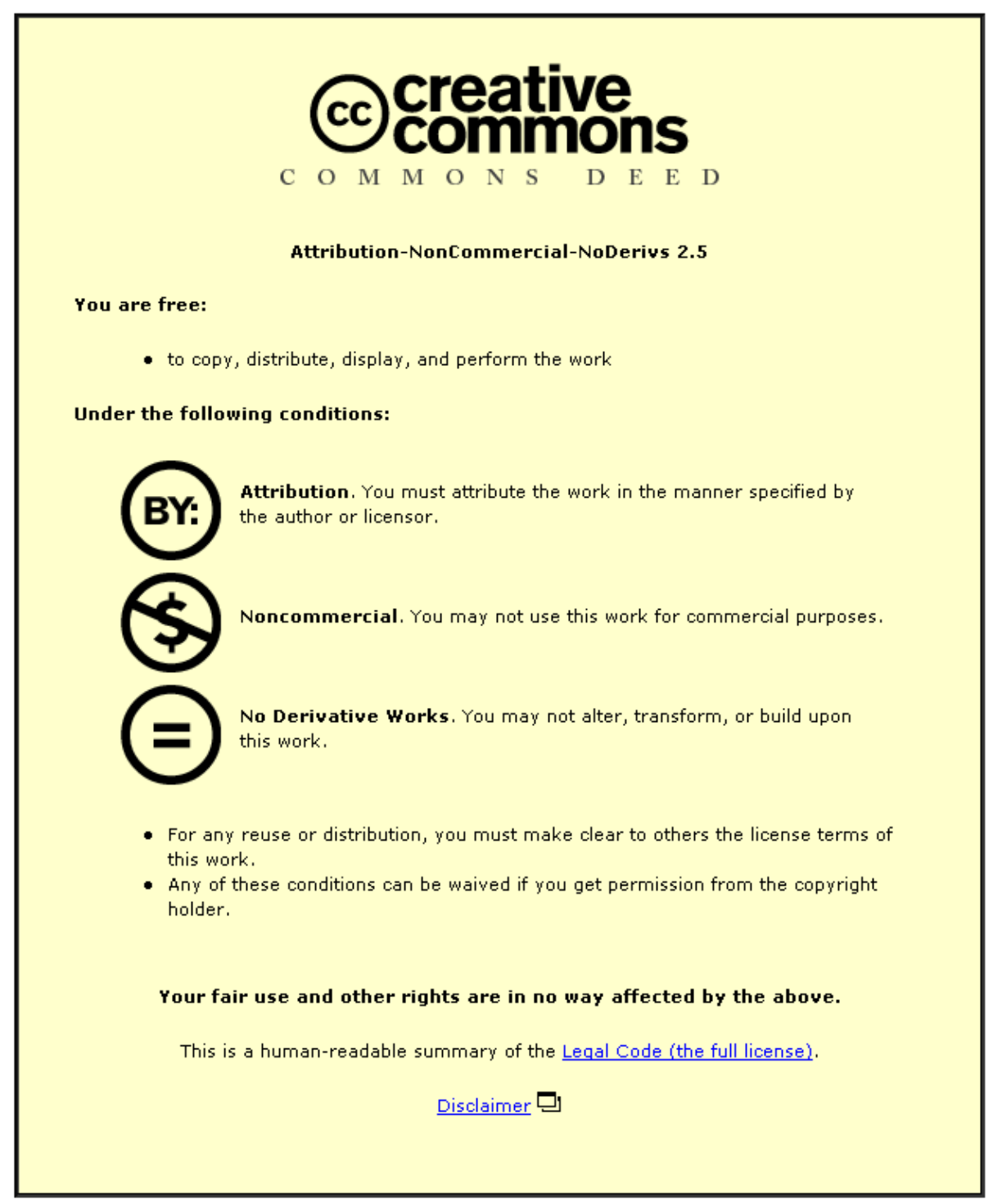

For the full text of this licence, please go to: http://creativecommons.org/licenses/by-nc-nd/2.5/ 


\title{
THE SIMULATION OF AERIAL MOVEMENT - I. THE DETERMINATION OF ORIENTATION ANGLES FROM FILM DATA
}

\author{
M. R. YEADON \\ Biomechanics Laboratory, Faculty of Physical Education, University of Calgary, Canada
}

\begin{abstract}
Quantitative mechanical analyses of human movement require the time histories of the angles which specify body configuration and orientation. When these angles are obtained from a filmed performance they may be used to evaluate the accuracy of a simulation model. This paper presents a method of determining orientation angles and their rates of change from film data. The stages used comprise the synchronisation of data obtained from two camera views, the determination of three-dimensional coordinates of joint centres, the calculation of an angle from a sequence of sine and cosine values and the curve fitting of angles using quintic splines. For each stage, other possible approaches are discussed. Original procedures are presented for obtaining individual error estimates of both the film data and the calculated angles to permit the automatic fitting of quintic splines for interpolation and differentiation and for deriving the time history of an angle as a continuous function from a sequence of sine and cosine values. The method is applied to a forward somersault with $1 \frac{1}{2}$ twists and the average error estimate of 17 orientation angles is obtained as 2.1 degrees.
\end{abstract}

\section{INTRODUCTION}

A quantitative three-dimensional mechanical analysis of human movement requires the time histories of the angles which specify body configuration and orientation. In the case of aerial movement the rotational motion is governed by the conservation of angular momentum equation and the first derivatives of angles are needed. This paper presents a method of determining these angles and their rates of change from film data and discusses other possible approaches.

For simple planar analyses of movement, angles may be determined by the use of a single cinecamera whose optical axis is normal to the plane of movement. For general three-dimensional body configurations, several cameras are needed, Noss (1967) stated that the angle between two lines may be determined as the average of the apparent angles given by three cameras with orthogonal optical axes. Spray (1973) and Putnam (1979) gave examples showing that the formula of Noss was incorrect. Walton (1981) observed that 'the expressions for which Noss was searching do exist, but they are more complex than a simple average'.

In fact, it is not possible to calculate the magnitude of an angle from the magnitudes of three projections. This can be shown by the following example. For simplicity consider the case in which the three cameras are situated at infinity on three orthogonal coordinate axes so that the apparent angles are the same as the orthogonal projections on the three coordinate planes. The four points $O(0,0,0)$, $A\left(1,1, \frac{1}{2}\right), B(1,1,1)$ and $C(1,1,2)$ define angles $B O A$ and $B O C$ (Fig. 1). These angles have equal projections on each of the three coordinate planes but are not equal to each other. Thus, a knowledge of the magnitudes of three projections of an angle is insufficient to determine the magnitude of the angle.

This indeterminacy arises because the expression for the angle between two lines involves four unknowns, namely two direction cosines for each line, whereas the magnitudes of three projections represents only three equations.

If the directions of the two lines are known, then it is a simple matter to calculate the angle between them. However, the description of the orientation of a body segment requires not only a means of calculating angles but also a system of reference frames relative to which orientation angles are defined. 


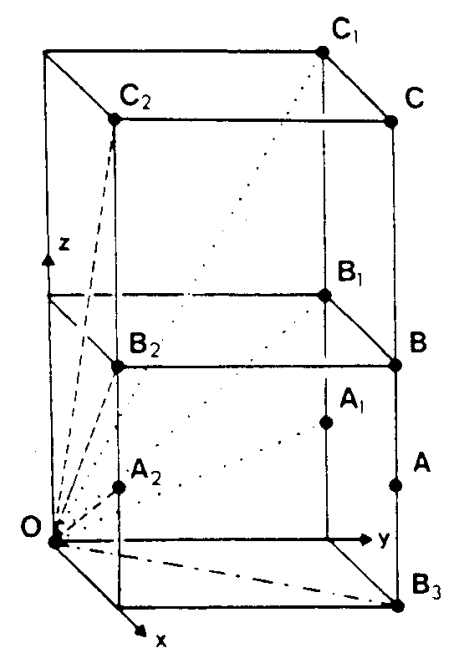

Figure 1: The projections $B_{1} O A_{1}, B_{2} O A_{2}, B_{3} O B_{3}$ of angle $B O A$ are equal to the projections $B_{1} O C_{1}$, $\mathrm{B}_{2} O \mathrm{C}_{2}, \mathrm{~B}_{3} O \mathrm{~B}_{3}$ of angle $\mathrm{BOC}$ whereas angles $\mathrm{BOA}$ and $\mathrm{BOC}$ are not equal.

\section{METHOD}

The various stages of the method are presented in a logical sequence. The system of orientation angles is defined and a technique is presented for determining the angles from the three-dimensional coordinates of body joint centres. The adoption of a quintic spline curve fitting technique for determining interpolated values and rates of change is discussed and this leads into the design of the film digitisation procedure.

\section{External orientation angles}

The whole body orientation in space, or external orientation. is defined as the orientation of a frame of reference $f$ which rotates with the body, relative to a non-rotating frame of reference $i$. The frame of reference $f$ is usually chosen to be fixed in one segment of the body (e.g. Ramey and Yang, 1981) but may be defined more generally using a number of segments (Yeadon, 1984). Frame $f$ comprises a right-handed triad $\left(\mathbf{f}_{1}, \mathbf{f}_{2}, \mathbf{f}_{3}\right)$ of mutually orthogonal unit vectors. Frame $i$ comprises the triad $\left(\mathbf{i}_{1}, \mathbf{i}_{2}, \mathbf{i}_{3}\right)$. The orientation of frame $f$ relative to frame $i$ is given by the angles $\phi, \theta$ and $\psi$ which are defined here in the following way.

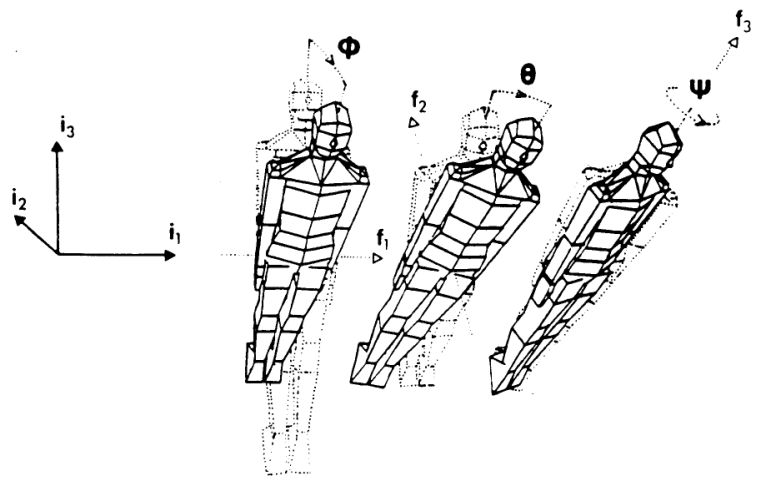

Figure 2: The angles $\phi, \theta$ and $\psi$, which correspond to somersault, tilt and twist, define the orientations of the body axes $\mathbf{f}_{1}, \mathbf{f}_{2}, \mathbf{f}_{3}$ relative to the non-rotating axes $\mathbf{i}_{1}, \mathbf{i}_{2}, \mathbf{i}_{3}$.

If frame $f$ is initially aligned with frame $i$ then successive rotations through $\phi$ about $\mathbf{f}_{1}, \theta$ about $\mathbf{f}_{2}$ and $\psi$ about $\mathbf{f}_{3}$ bring frame $f$ into its final orientation. For aerial movement the angles $\phi, \theta$ and $\psi$ 
correspond to somersault, tilt and twist (Fig. 2). This choice of angles was adopted by Passerello and Huston (1971) and Van Gheluwe (1981), but there are a number of other systems which may be used (Goldstein, 1950).

If the directions of the vectors $\mathbf{i}_{1}, \mathbf{i}_{2}, \mathbf{i}_{3}, \mathbf{f}_{1}, \mathbf{f}_{2}, \mathbf{f}_{3}$ are known then the angles $\phi, \theta, \psi$ may be determined as follows.

Let $S_{f i}$ be the matrix which transforms the $f$-coordinates of a free vector into $i$-coordinates, i.e., $S_{f i}[\mathbf{v}]_{f}=[\mathbf{v}]_{i}$ where $[\mathbf{v}]_{f}$ and $[\mathbf{v}]_{i}$ are the column vector representations of the vector $\mathbf{v}$ in frames $f$ and $i$. Frame $f$ will be brought into alignment with frame $i$ by successive rotations through $-\psi$ about $\mathbf{f}_{3},-\theta$ about $\mathbf{f}_{2}$ and $-\phi$ about $\mathbf{f}_{1}$. Let $R_{3}(-\psi), R_{2}(-\theta)$ and $R_{1}(-\phi)$ be the rotation matrices corresponding to the successive rotations. The columns of these rotation matrices are merely the new coordinates of the previous directions of the unit vectors $\mathbf{f}_{1}, \mathbf{f}_{2}$ and $\mathbf{f}_{3}$.

Thus:

$$
\begin{aligned}
R_{1}(-\phi) & =\left[\begin{array}{ccc}
1 & 0 & 0 \\
0 & c \phi & -s \phi \\
0 & s \phi & c \phi
\end{array}\right] \\
R_{2}(-\theta) & =\left[\begin{array}{ccc}
c \theta & 0 & s \theta \\
0 & 1 & 0 \\
-s \theta & 0 & c \theta
\end{array}\right] \\
R_{3}(-\psi) & =\left[\begin{array}{ccc}
c \psi & -s \psi & 0 \\
s \psi & c \psi & 0 \\
0 & 0 & 1
\end{array}\right]
\end{aligned}
$$

where $c$ denotes cosine and $s$ denotes sine.

Similarly, the columns of the matrix $S_{f i}$ are the components in frame $i$ of the unit vectors $\mathbf{f}_{1}, \mathbf{f}_{2}, \mathbf{f}_{3}$. Thus:

$$
S_{f i}=\left[\begin{array}{lll}
F 1(1) & F 2(1) & F 3(1) \\
F 1(2) & F 2(2) & F 3(2) \\
F 1(3) & F 3(2) & F 3(3)
\end{array}\right]
$$

where $F k(j)$ denotes the $j$ th component of $(f)_{k}$ in frame $i$.

$S_{f i}$ may also be evaluated as:

$$
S_{f i}=R_{1}(-\phi) \cdot R_{2}(-\theta) \cdot R_{3}(-\psi) .
$$

Thus:

$$
S_{f i}=\left[\begin{array}{ccc}
c \theta c \psi & -c \theta s \psi & s \theta \\
c \phi s \psi+s \theta s \theta c \psi & c \theta c \psi-s \phi s \theta s \psi & -s \phi c \theta \\
s \phi s \psi-c \phi s \theta c \psi & s \phi c \psi+c \phi s \theta s \psi & c \phi c \theta
\end{array}\right] .
$$

Comparing corresponding elements in the two expressions for $S_{f i}$ permits the calculation of the sine and cosine of each orientation angle as:

$$
\begin{aligned}
s \theta & =F 3(1) \\
c \theta & =\left(1-s^{2} \theta\right)^{\frac{1}{2}} \\
s \phi & =-F 3(2) / c \theta \\
c \phi & =F 3(3) / c \theta \\
s \psi & =-F 2(1) / c \theta \\
c \psi & =F 1(1) / c \theta .
\end{aligned}
$$

Since the cosine of angle $\theta$ is defined as being positive by the equation $c \theta=\left(1-s^{2} \theta\right)^{\frac{1}{2}}, \theta$ will lie in the range $-\frac{1}{2} \pi<0 \leq \frac{1}{2} \pi$. At $\theta=\frac{1}{2} \pi$ there is a singularity in the sense that $\phi$ and $\psi$ are indeterminate. For aerial movements such as twisting somersaults this will not present problems since the tilt angle $\theta$ typically lies in the range $-\pi / 8<\theta<\pi / 8$ and does not approach the value $\frac{1}{2} \pi$. 


\section{Internal orientation angles}

The configuration of the body, or internal orientation, is defined by angles which specify the relative orientations of adjacent body segments. It is assumed that these segments are rigid bodies and that relative movement of adjacent segments comprises rotation about joint centres which are axed in the rigid bodies. As an example, the orientation of the left upper arm relative to the chest will be defined.

Figure 3 shows the joint centres S, E and W at the left shoulder, elbow and wrist. The coordinate frame a of the left upper arm comprises unit vectors $\mathbf{a}_{1}, \mathbf{a}_{2}, \mathbf{a}_{3}$ where:

$$
\begin{aligned}
& \mathbf{a}_{3} \text { is parallel to } \overline{\mathrm{ES}} \\
& \mathbf{a}_{2} \text { is parallel to } \mathbf{a}_{3} \times \overline{\mathrm{WE}} \\
& \mathbf{a}_{1}=\mathbf{a}_{2} \times \mathbf{a}_{3} .
\end{aligned}
$$

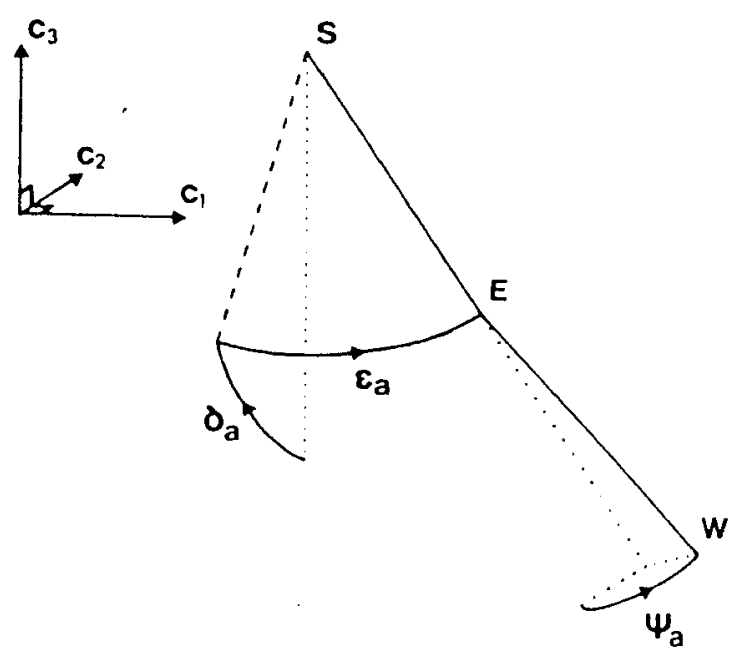

Figure 3: The positions of the joint centres S, E and W at the left shoulder, elbow and wrist determine the angles $\delta_{a}, \varepsilon_{a}$ and $\psi_{a}$ which define the orientation of the left upper arm relative to the chest axes $\mathbf{c}_{1}, \mathbf{c}_{2}$ and $\mathbf{c}_{3}$.

If the arm frame $a$ is initially aligned with the chest frame $c$ then successive rotations through $-\delta_{a}$ about $\mathbf{a}_{1},-\varepsilon_{a}$ about $\mathbf{a}_{2}, \psi_{a}$ about $\mathbf{a}_{3}$, bring frame $a$ into its final orientation (Fig. 3 ). For this choice of rotations $\delta_{a}$ is the angle of elevation in the sagittal plane, $\varepsilon_{a}$ is the angle of abduction away from the sagittal plane and $\psi_{a}$ is the angle of medio-lateral rotation about the longitudinal axis of the left upper arm.

Let $S_{a c}$ be the matrix which transforms the $a$-coordinates of a free vector into $c$-coordinates. It should be noted that the origins of frames $a$ and $c$ are not of importance as far as rotations of a free vector are concerned. The rotations of the left upper arm will, of course, be about the joint centre $\mathrm{S}$ at the left shoulder.

Frame $a$ will be brought into alignment with frame $c$ by successive rotations through $-\psi_{a}$ about $\mathbf{a}_{3}$, $\varepsilon_{a}$ about $\mathbf{a}_{2}$ and $\delta_{a}$ about $\mathbf{a}_{1}$. If $R_{3}\left(-\psi_{a}\right), R_{2}\left(\varepsilon_{a}\right)$ and $R_{1}\left(\delta_{a}\right)$ are the corresponding rotation matrices:

$$
\begin{aligned}
S_{a c} & =R_{1}\left(\delta_{a}\right) \cdot R_{2}\left(\varepsilon_{a}\right) \cdot R_{3}\left(-\psi_{a}\right) \\
& =\left[\begin{array}{ccc}
c \varepsilon c \psi & -c \varepsilon s \psi & -s \varepsilon \\
s \delta s \varepsilon c \psi+c \delta s \psi & -s \delta s \varepsilon s \psi+c \delta c \psi & s \delta c \varepsilon \\
c \delta s \varepsilon c \psi-s \delta s \psi & -c \delta s \varepsilon c \psi-s \delta c \psi & c \delta c \varepsilon
\end{array}\right]
\end{aligned}
$$

where $s$ denotes sine and $c$ denotes cosine.

The columns of $S_{a c}$ are the $c$-coordinates of the unit vectors $\mathbf{a}_{1}, \mathbf{a}_{2}, \mathbf{a}_{3}$ so that:

$$
S_{a c}=\left[\begin{array}{lll}
A 1(1) & A 2(1) & A 3(1) \\
A 1(2) & A 2(2) & A 3(2) \\
A 1(3) & A 2(3) & A 3(3)
\end{array}\right]
$$


where $A k(j)$ denotes $j$ th component of $\mathbf{a}_{k}$ in frame $c . A k(j)$ may be calculated as the scalar product of the vectors $\mathbf{a}_{k}$ and $\mathbf{c}_{j}(k=1,2,3 ; j=1,2,3)$.

Comparing corresponding elements in the two expressions for $S_{a c}$ permits the calculation of the sine and cosine of each orientation angle as:

$$
\begin{aligned}
\sin \varepsilon_{a} & =-A 3(1) \\
\cos \varepsilon_{a} & =\left(1-\sin ^{2} \varepsilon_{a}\right)^{1 / 2} \\
\sin \delta_{a} & =A 3(2) / \cos \varepsilon_{a} \\
\cos \delta_{a} & =A 3(3) / \cos \varepsilon_{a} \\
\sin \psi_{a} & =-A 2(1) / \cos \varepsilon_{a} \\
\cos \psi_{a} & =A 1(1) / \cos \varepsilon_{a} .
\end{aligned}
$$

Since the cosine of angle $\varepsilon_{a}$ is defined to be positive, $\varepsilon_{a}$. will lie in the range $-\frac{1}{2} \pi<\varepsilon_{a} \leq \frac{1}{2} \pi$. There is a singularity at $\varepsilon_{a}=\frac{1}{2} \pi$ in the sense that $\delta_{a}$ and $\psi_{a}$ are indeterminate when $\cos \varepsilon_{a}=0$. This itself is not a problem since, when film data are used, $\cos \varepsilon_{a}$ will never be exactly zero. When the arm is abducted so that it is approximately perpendicular to the sagittal plane, $\cos \varepsilon_{a}$ will be small and errors in the film data may produce large changes in the angles $\varepsilon_{a}$ and $\psi_{a}$. Again this is not a problem since the arm positions corresponding to the erroneous angle values will always be close to the actual arm positions and the angular momenta associated with the erroneous arm movements will be small.

However, if repeated digitisation of the film data is used to produce more than one estimate of each angle, it is possible for two estimates to diverge and differ by approximately $2 \pi$ radians. This situation may be avoided by replacing a given estimate with the average of that estimate and the first estimate whenever the difference from the first estimate is greater than $\pi$ radians. This procedure forces all estimates to follow the same general pattern as the first estimate. An average of all estimates may then be used.

It should be noted that singularity problems may be avoided by using attitude matrices (Woltring and Huiskes, 1985) or Eulerian parameters (Huston et al., 1978) rather than orientation angles. The present approach was adopted so that orientation angles could be used as input to a simulation model (Yeadon et al., 1990).

\section{Calculation of an angle from sine and cosine values}

Once the sine and cosine of an angle $A$ are known it is a simple matter to determine the angle in a particular range: e.g. $-\pi<A \leq \pi$. However, it is necessary to determine a sequence of angle values from a sequence of sine and cosine values in such a way that the time history of the angle is continuous. This may be done as follows.

The initial value $A_{0}$ is obtained in the range $-\pi / 2<A \leq 3 \pi / 2$ from the values $\sin A_{0}$ and $\cos A_{0}$ using:

$$
\begin{aligned}
& A_{0}=\arctan \left(\sin A_{0} / \cos A_{0}\right)\left(\text { for } \cos A_{0}>0\right) \\
& A_{0}=\pi+\arctan \left(\sin A_{0} / \cos A_{0}\right)\left(\text { for } \cos A_{0} \leq 0\right)
\end{aligned}
$$

Subsequent values are calculated inductively. In order to calculate the value of angle $A$ from $\sin A$, $\cos A$ and the preceding value $A_{1}$, let $B$ be the angle in the range $-\pi<B \leq \pi$ defined by:

$$
\begin{aligned}
& \cos B=\cos A \cos A_{1}+\sin A \sin A_{1}=\cos \left(A-A_{1}\right) \\
& \sin B=\sin A \cos A_{1}-\cos A \sin A_{1}=\sin \left(A-A_{1}\right) .
\end{aligned}
$$

$A$ is then obtained as $A=A_{1}+B$ so that $A$ differs from the preceding value $A_{1}$ by less than $\pi$ radians. This incremental procedure avoids the discontinuities which arise when an angle is calculated solely from its sine and/or cosine as was done by Gervais and Marino (1983).

\section{Curve fitting techniques}

Once a sequence of angle values has been determined, it is necessary to obtain angle values and rates of change at intermediate times in order to integrate the equations of motion using a numerical procedure. 
The determination of interpolated values and derivatives may be accomplished by fitting a 'smooth' function to the data values. Functions which have been used are polynomials (Plagenhoef, 1968), Fourier series (Anderssen and Bloomfield, 1974; Hatze, 1981), cubic splines (Reinsch, 1967) and quintic splines (Wood and Jennings, 1979). Cubic splines have been shown to give better estimates of derivatives than polynomials (Zernicke et al., 1976) while quintic splines produce better endpoint derivatives than cubic splines and Fourier series (Wood and Jennings, 1979).

The closeness of fit and smoothness of a quintic spline are governed by error estimates of the data. In the quintic spline of Woltring (1985), adapted from Utreras (1980), a single estimate of accuracy is inferred from the data sets whereas the spline of Wood and Jennings (1979) permits the weighting of data points by requiring individual error estimates for each data value.

The latter procedure finds the quintic spline function $g(t)$ which minimises the smoothness integral:

$$
\int_{t_{1}}^{t_{N}}\left[g^{\prime \prime \prime}(t)\right]^{2} \mathrm{~d} t \text { (where } g^{\prime \prime \prime} \text { is the third derivative of } g \text { ) }
$$

subject to the closeness of fit constraint:

$$
\sum_{i=1}^{N}\left(g\left(t_{i}\right)-y_{i}\right)^{2} /\left(\delta y_{i}\right)^{2} \leq N
$$

where $\left\{\left(t_{i}, y_{i}\right), i=1, N\right\}$ is the set of data points and $\delta y_{i}$ are standard errors of measurement.

This quintic spline procedure was adopted since the constraint condition permits the use of individual error estimates at each data point whereas the spline of Utreras assumes that errors are normally distributed and uses a single error estimate. When digitising film of twisting somersaults it is clear that there will be phases in which a given joint centre cannot be seen so that an estimate of its location will be poor. As a consequence, the accuracy of angle estimates will be variable and it is appropriate to use individual error estimates at each data value. Such individual error estimates may be obtained from film by using repeated digitisation to produce two or more estimates of each angle value.

It is possible that the general spline package of Lyche and Schumaker (1973), expanded by Woltring (1986), will provide better derivative estimates than the quintic spline of Wood and Jennings (1979) since the package permits higher order splines and estimates an optimal amount of smoothing.

\section{Cinematography}

Three-dimensional filming techniques may be divided into two groups:

(1) Systems which require field measurements (Miller, 1970; Bergemann, 1974; Penrose et al., 1976).

(2) Systems which do not require field measurements (Van Gheluwe, 1978; Shapiro, 1978; Walton, 1981).

Group 1 systems have the disadvantage that either the cameras must be placed in known locations or on-site measurements must be made to determine the camera locations. Group 2 systems are free from such camera location requirements but require that the reference points used for calibration be distributed throughout the region of interest (Miller et al., 1980; Wood and Marshall, 1986). Since both techniques produce accuracies of $5 \mathrm{~mm}$ in a field of $3 \mathrm{~m}$ (Penrose et al., 1976; Shapiro, 1978) a choice of system may be made on the basis of convenience.

In the present study trampoline movements were to be filmed. In this situation it was easier to place the cameras in specified locations rather than to locate reference points throughout the field of movement. The following field measurement approach was therefore adopted.

Cameras $\mathrm{F}$ and $\mathrm{S}$ are positioned to the front and to the side of a tri-axial calibration frame with origin $\mathrm{O}$ so that $\overline{\mathrm{FO}}$ and $\overline{\mathrm{SO}}$ are horizontal and perpendicular (Fig. 4). The distances $d \mathrm{FO}$ and $d \mathrm{SO}$ are measured. The cameras are aligned so that the origin $\mathrm{O}$ is approximately centred in the camera viewfinders. The actual orientation of each camera is determined by digitising the four corners of the film frame so that the position of the centre of the image relative to the image of the reference origin $\mathrm{O}$ can be obtained (Yeadon, 1984).

Knowing the location and orientation of each camera and the digitised coordinates of the points of the calibration frame permits the reconstruction of rays $\overline{\mathrm{FA}}$ and $\overline{\mathrm{SB}}$, on which the joint centre $\mathrm{R}$ should lie, from the digitised coordinates of the film images of $\mathrm{R}$ (Fig. 4). An estimate of the location of point $\mathrm{R}$ 


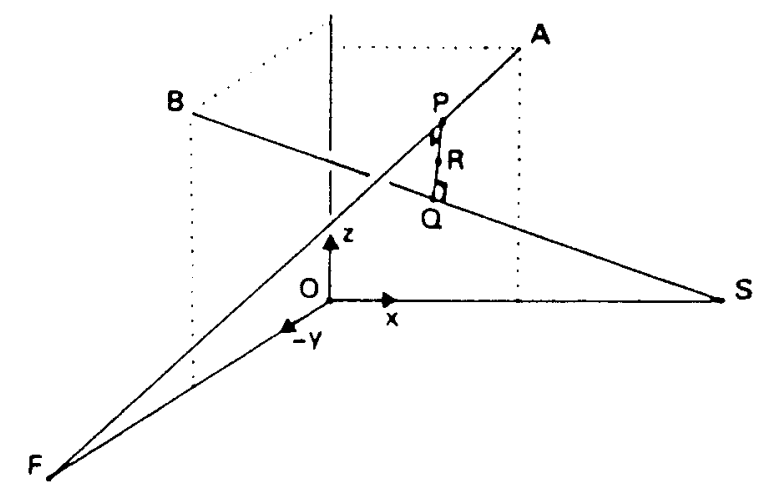

Figure 4: The location of the point $\mathrm{R}$ in space is reconstructed from film data as the midpoint of the common perpendicular $\overline{\mathrm{PQ}}$ to the rays $\overline{\mathrm{FA}}$ and $\overline{\mathrm{SB}}$ from the camera positions $\mathrm{F}$ and $\mathrm{S}$.

is then obtained as the midpoint of the common perpendicular $\overline{\mathrm{PQ}}$ to the rays $\overline{\mathrm{FA}}$ and $\overline{\mathrm{SB}}$. This location minimises the sum of the squares of the distances from the rays $\overline{\mathrm{FA}}$ and $\overline{\mathrm{SB}}$.

In this way the three-dimensional locations of the joint centres are obtained so that the unit vectors defining the frames of reference of the body segments may be determined.

\section{Synchronisation}

Data sets obtained from two or more views may be combined to produce accurate estimates of spatial coordinates only when they correspond to the same instant of time. Such synchronisation may be achieved either physically, where the original data sets are obtained at the same times, or analytically, where time-matched data sets are interpolated from the original data.

Physical synchronisation may be achieved by using a single camera in conjunction with a mirror, although a large mirror is required for human movement and distortion errors can occur. Another method is to use electronically phase-locked cine cameras. Redlake Corporation states that the accuracy of LOCAM phase lock camera shutters is \pm 9 degrees which corresponds to a difference of $\pm 0.25 \mathrm{~ms}$ at 100 frames per second.

Analytical synchronisation may be effected using a timing device which is in view of each camera and then fitting the time-displacement data points with a suitable function to obtain interpolated values. The accuracy of the time values for a millisecond timer is $\pm 0.5 \mathrm{~ms}$, but this can be improved by fitting a straight line to the frame times to remove the effect of rounding the times to the nearest millisecond. This procedure assumes that the camera framing rates remain constant. In this study, spring driven cameras were used and so the cubic spline of Reinsch (1967) was used to fit the frame times, since it allows for variation in the framing rate but defaults to a linear fit when apparent variation can be accounted for by rounding errors.

While it is possible to use an interpolation spline, which passes through the time-displacement data points, to obtain interpolated values, such a procedure will maintain or increase the error level present in the displacement data. A better procedure is to reduce the error level of the interpolated estimates by fitting a spline whose closeness of fit is based upon the errors in the displacement data.

\section{Error estimates}

In order to fit splines to the frame times, displacement data and orientation angles, it is necessary to have estimates of the errors in the data values.

If the actual time $t$ is rounded to the nearest millisecond to give the estimate $T$, the error $(t-T)$ has variance $v$ where:

$$
v=\int_{T-\frac{1}{2}}^{T+\frac{1}{2}}(t-T)^{2} \mathrm{~d} t=\int_{-\frac{1}{2}}^{+\frac{1}{2}} t^{2} \mathrm{~d} t=\left[\frac{t^{3}}{3}\right]_{-\frac{1}{2}}^{+\frac{1}{2}}=\frac{1}{12} .
$$

Thus, the standard deviation is $1 / \sqrt{ } 12 \cong 0.3 \mathrm{~ms}$. This value may be used as an error estimate when spline fitting the frame times. 
If each film is digitised $n$ times there will be $n$ independent estimates $x_{i}(i=1, n)$ of each displacement data value $x$. If $x_{i}(i=1, n)$ are normally distributed then an unbiased estimate of the variance $\operatorname{var}(x)$ is given by:

$$
\operatorname{var}(x)=\sum_{1}^{n}\left(x_{i}-a\right)^{2} /(n-1)
$$

where $a=\sum_{1}^{n} x_{i} / n$ is the mean.

In the present study, the number of digitisations was minimised by choosing $n=2$ so that a local estimate of the variance is given by:

$$
V_{l}=\frac{1}{2}\left(x_{1}-x_{2}\right)^{2}
$$

In order to calculate a standard deviation of the estimates $x_{1}$ and $x_{2}$ for fitting splines, there are two obvious possibilities. The first is to use the expression for the local variance $V_{l}$ which is based solely on the difference $\left(x_{1}-x_{2}\right)$. The second possibility is to calculate a global variance $V_{g}$ over all the film frames. The disadvantage of the first method is that points will be overweighted in the spline procedure when the difference $\left(x_{1}-x_{2}\right)$ chances to be artificially low. The disadvantage of the global technique is that no use is made of the local information given by $\left(x_{1}-x_{2}\right)$.

A compromise solution may be obtained by defining the error estimate as: $\delta_{x}=\left(k V_{l}+(1-k) V_{g}\right)^{1 / 2}$ with $0<k<1$. A choice of $k=0.25$ means that a large error is underestimated by at most a factor of 2 and a lower bound of about $0.87 V_{g}^{1 / 2}$ is placed on $\delta_{x}$.

Using two cameras and digitising each film twice produces four combinations of the interpolated data values. As a consequence, there are four estimates $A_{11}, A_{12}, A_{21}, A_{22}$ of each orientation angle, where $A_{i j}$ is produced by the $i$ th digitisation of the front camera film and the $j$ th digitisation of the side camera film $(i=1,2 ; j=1,2)$. Thus. there are two independent pairs of angle estimates, namely $A_{11}: A_{22}$ and $A_{12}: A_{21}$.

Each orientation angle $A$ is some function $G$ of the digitised coordinates of all the joint centres:

$$
A=G\left(x_{1}, x_{2}, \ldots x_{n}, y_{1}, y_{2} \ldots y_{n}\right)
$$

where $x_{i}(i=1, n)$ are the coordinates obtained using the first camera and $y_{i}(i=1, n)$ are the coordinates obtained using the second camera.

If second degree terms in the digitisation errors $\delta x_{i}, \delta y_{i}$ are neglected, the Taylor expansion becomes:

$$
\begin{aligned}
A & =G\left(a_{1}, a_{2}, \ldots a_{n}, b_{1}, b_{2}, \ldots b_{n}\right) \\
& +\sum_{1}^{n} \frac{\delta G}{\delta x_{i}} \cdot \delta x_{i}+\sum_{1}^{n} \frac{\delta G}{\delta y_{i}} \cdot \delta y_{i}
\end{aligned}
$$

where $a_{i}$ and $b_{i}$ are the error-free values of the digitised coordinates, $x_{i}=a_{i}+\delta x_{i}$ and $y_{i}=b_{i}+\delta y_{i}$.

Thus:

$$
A=A_{0}+X+Y
$$

where $A_{0}$ is the error-free value of angle $A, X$ is a linear function of the $\delta x_{i}$ digitising errors and $Y$ is a linear function of the $\delta y_{i}$ digitising errors.

The four estimates of angle $A$ take the form:

$$
\begin{aligned}
& A_{11}=A_{0}+X_{1}+Y_{1} \\
& A_{12}=A_{0}+X_{1}+Y_{2} \\
& A_{21}=A_{0}+X_{2}+Y_{1} \\
& A_{22}=A_{0}+X_{2}+Y_{2}
\end{aligned}
$$

where $X_{1}, Y_{1}$ are errors arising from the first digitisations and $X_{2}, Y_{2}$ are errors arising from the second digitisations.

Thus, the mean $\bar{A}$ of the four estimates is given by:

$$
\bar{A}=A_{0}+\frac{1}{2}\left(X_{1}+X_{2}\right)+\frac{1}{2}\left(Y_{1}+Y_{2}\right)
$$


where $X_{1}$ and $X_{2}$ are values taken by the random variable $X$ and $Y_{1}$ and $Y_{2}$ are values taken by the random variable $Y$. For reasons of symmetry the mean $\bar{A}$ is the best estimate of the error-free value $A_{0}$.

The variance $\operatorname{var}(\bar{A})$ of the mean $\bar{A}$ is given by:

$$
\begin{aligned}
\operatorname{var}(\bar{A}) & =\frac{1}{2} \operatorname{var}(X)+\frac{1}{2} \operatorname{var}(Y) \\
& =\frac{1}{2}\left[\frac{1}{2}\left(X_{1}-X_{2}\right)^{2}\right]+\frac{1}{2}\left[\frac{1}{2}\left(Y_{1}-Y_{2}\right)^{2}\right] \\
& =\left[\left(A_{11}-A_{22}\right)^{2}+\left(A_{12}-A_{21}\right)^{2}\right] / 8 .
\end{aligned}
$$

A quintic spline may now be fitted to the average $A$ to obtain first and second derivatives. If error estimates of the derivatives are required, these may be obtained by fitting individual quintic splines to $A_{11}, A_{12}, A_{21}$ and $A_{22}$.

\section{APPLICATION}

The method was applied to a performance of a forward somersault with $1 \frac{1}{2}$ twists on trampoline. Bolex $16 \mathrm{~mm}$ spring driven cameras were positioned to the front and to the side of the trampoline forming a right-angled triangle with a tri-axial calibration frame which was inserted through the centre of the trampoline bed. The distances of the cameras from the origin of the calibration frame were measured, the calibration frame was filmed and was then removed from the trampoline. A millisecond timer was placed in the fields of view of the two cameras and the twisting somersault was filmed using nominal framing rates of 64 frames per second.
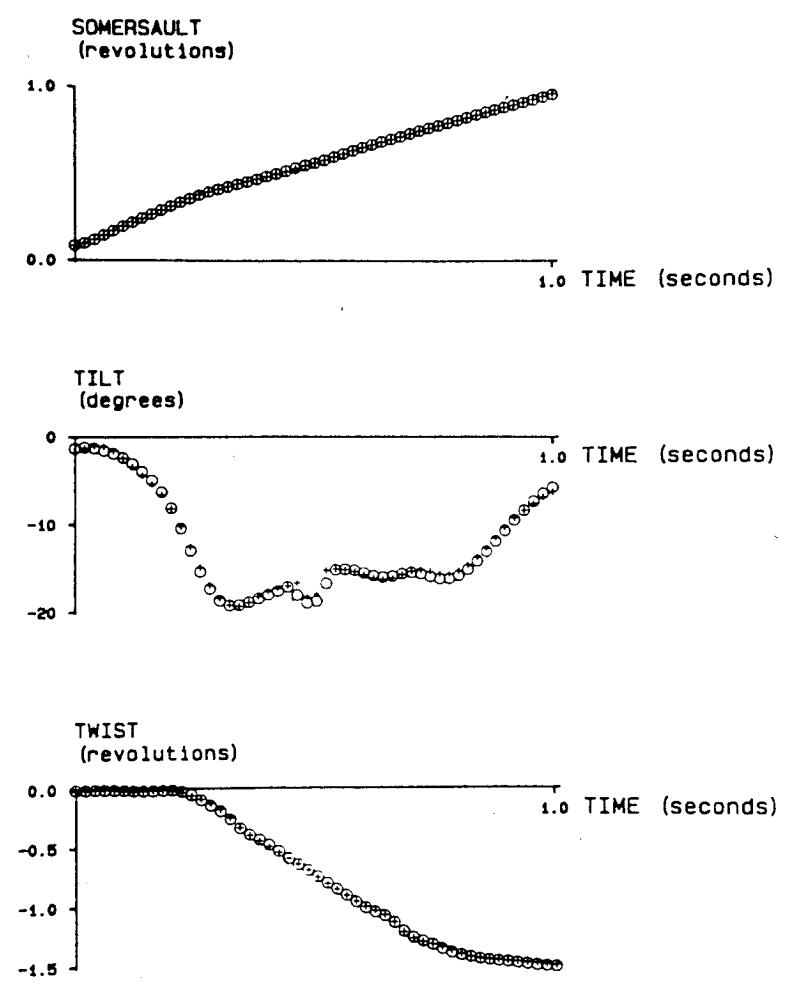

Figure 5: The time histories of the angles of somersault, tilt and twist for a forward somersault with $1 \frac{1}{2}$ twists. Two independent sets of angle estimates, obtained by repeated film digitisation, are represented using $(+)$ and $(\circ)$.

The images of the calibration points and the four corners of the projected film frame were digitised to establish scale factors and camera orientation. This was repeated four times in order to reduce the 
a
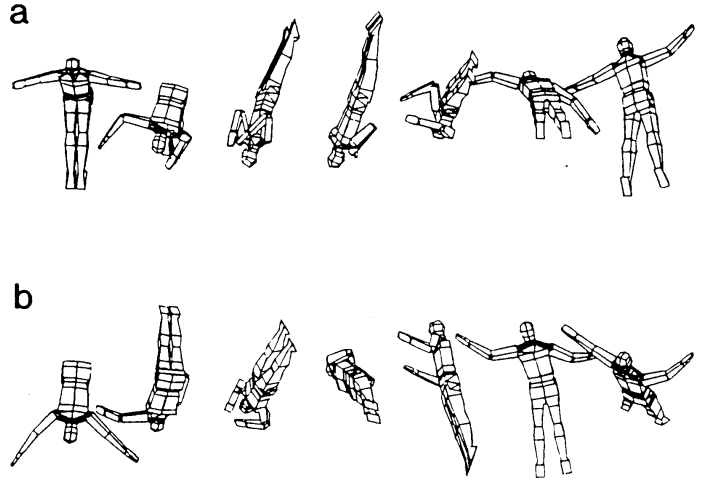

Figure 6: Graphics sequences of a forward somersault with $1 \frac{1}{2}$ twists viewed from (a) in front and (b) above.

effects of digitisation errors. Joint centres at the wrist, elbow, shoulder, hip, knee and ankle on the left and right sides of the body were digitised for each frame of the airborne phase. The time given by the millisecond timer was obtained for each film frame. The above digitisation procedure was performed twice for each of the two films.

The time values for each frame were corrected using a cubic spline to remove rounding errors. Each set of displacement data was fitted with a quintic spline, using error estimates based upon the repeated digitisation, and interpolated values were obtained at 100 equally spaced time intervals to produce synchronised sets of displacement data.

Three-dimensional coordinates of the joint centres were calculated at each of the 101 time values. Since each elm was digitised twice, there were four combinations of the digitised data so that four estimates were obtained for the location of each joint centre. This produced four estimates of each orientation angle at each time value. The averages of the four estimates were fitted with quintic splines using error estimates based upon the variance of the four estimates.

Figure 5 depicts the time histories of two independent estimates of each of the angles $\phi, \theta$ and $\psi$ which correspond to somersault, tilt and twist. The mean error estimates for these three angles were $0.2^{\circ}$ for somersault, $0.2^{\circ}$ for tilt and $1.1^{\circ}$ for twist. The mean value of the error estimates for all 17 orientation angles was $2.1^{\circ}$. These values can be regarded as underestimates since they are based upon repeated digitisation and do not include any systematic errors.

The calculated orientation angles were input into the SAMMIE man model (Kingsley et al., 1981) to produce sequences depicting the twisting somersault from two viewpoints. Figure 6 a gives the view from the front camera position whereas the overhead view shown in Fig. 6b corresponds to neither camera viewpoint. The ability to provide viewpoints which are not directly available can be useful for gaining a better appreciation of the changes in orientation and configuration during a movement.

The quintic splines of the orientation angles may be used to obtain derivatives at any time. The accuracies of such derivative values will be considered in parts III and IV of this series where the calculation of angular momentum and the simulation of aerial movement are described (Yeadon, 1990; Yeadon et al., 1990).

\section{References}

Anderssen, R. S. and Bloomfield, P. (1974). A time series approach to numerical differentiation. Technometrics, 16:69-75.

Bergemann, B. W. (1974). Three-dimensional cinematography: a flexible approach. Research Quarterly, 45:302-309.

Gervais, P. and Marino, G. W. (1983). A procedure for determining angular positional data relative to the principal axes of the human body. Journal of Biomechanics, 16:109-113.

Goldstein, H. (1950). Classical Mechanics, page 108. Addison-Wesley, London. 
Hatze, H. (1981). The use of optimally regularised Fourier series for estimating higher-order derivatives of noisy biomechanical data. Journal of Biomechanics, 14:13-18.

Huston, R. L., Passerello, C. E., and Harlow, M. W. (1978). Dynamics of multirigid-body systems. Journal of Applied Mechanics, 45:889-894.

Kingsley, E. C., Schofield, N. A., and Case, K. (1981). SAMMIE: a computer aid for man machine modelling. Computer Graphics, 15:163-169.

Lyche, T. and Schumaker, L. (1973). Computation of smoothing and interpolating natural splines via local bases. SIAM Journal of Numerical Analysis, 10:1027-1038.

Miller, D. I. (1970). A computer simulation model of the airborne phase of diving. PhD thesis, Pennsylvania State University.

Miller, N. R., Shapiro, R., and McLaughlin, T. M. (1980). A technique for obtaining spatial kinematic parameters of segments of biomechanical systems from cinematographic data. Journal of Biomechanics, $13: 535-547$.

Noss, J. (1967). Control of photographic perspective in motion analysis. Journal of Health Physical Education Research, 38:81-84.

Passerello, C. E. and Huston, R. L. (1971). Human attitude control. Journal of Biomechanics, 4:95-102.

Penrose, T., Wood, G., and Blanksby, B. (1976). The accuracy of positional data in triaxial cinematography. Australian Journal of Health Physical Education Research, 71:7-12.

Plagenhoef, S. C. (1968). Computer programs for obtaining kinetic data on human movement. Journal of Biomechanics, 1:221-234.

Putnam, C. A. (1979). The triaxial cinematographic method of angular measurement. Research Quarterly, 50:140-143.

Ramey, M. R. and Yang, A. T. (1981). A simulation procedure for human motion studies. Journal of Biomechanics, 14:203-213.

Reinsch, C. H. (1967). Smoothing by spline functions. Numerical Mathematics, 10:177-183.

Shapiro, R. (1978). Direct linear transformation method for three-dimensional cinematography. Research Quarterly, 49:197-205.

Spray, J. (1973). Three-dimensional film data procedures: a vector approach. Master's thesis, University of Arizona.

Utreras, F. (1980). Un paquete de programas para ajustas curvas mediante functiones spline. Informe Tecnio MA80-8-209, Departamento de Matematicas, Faculdad de Ciencias Fisicas y Matematicas, Universidad de Chile, Santiago.

Van Gheluwe, B. (1978). Computerized three-dimensional cinematography for any arbitrary camera set-up. In Asmussen, E. and Jorgensen, K., editors, Biomechanics VI, pages 343-348, Baltimore. University Park Press.

Van Gheluwe, B. (1981). Computer simulation of an airborne backward twist somersault. In Morecki, A., Fidelus, K., K., K., and Wit, A., editors, Biomechanics VII-A, pages 200-205, Baltimore. University Park Press.

Walton, J. S. (1981). Close-range cine-photogrammetry: a generalised technique for quantifying gross human motion. PhD thesis, Pennsylvania State University.

Woltring, H. J. (1985). On optimal smoothing and derivative estimation from noisy displacement data in biomechanics. Human Movement Science, 4:229-245. 
Woltring, H. J. (1986). A Fortran package for generalised cross validatory spline smoothing and differentiation. Advanced Engineering Software, 8:104-107.

Woltring, H. J. and Huiskes, R. (1985). A statistically motivated approach to instantaneous helical axis estimation from noisy, sampled landmark coordinates. In Winter, D. A. et al., editors, Biomechanics $I X-B$, pages 274-279, Champaign. Human Kinetics Publishers.

Wood, G. A. and Jennings, L. S. (1979). On the use of spline functions for data smoothing. Journal of Biomechanics, 12:477-479.

Wood, G. A. and Marshall, R. N. (1986). The accuracy of dlt extrapolation in three-dimensional film analysis. Journal of Biomechanics, 19:781-785.

Yeadon, M. R. (1984). The mechanics of twisting somersaults. PhD thesis, Loughborough University.

Yeadon, M. R. (1990). The simulation of aerial movement - III. The determination of the angular momentum of the human body. Journal of Biomechanics, 23:75-83.

Yeadon, M. R., Atha, J., and Hales, F. D. (1990). The simulation of aerial movement - IV. A computer simulation model. Journal of Biomechanics, 23:85-89.

Zernicke, R. F., Caldwell, G., and Roberts, E. M. (1976). Fitting biomechanical data with cubic spline functions. Research Quarterly, 47:9-20. 